\title{
Role of an order-disorder phase transition in increasing the exchange magnetic field in a diluted magnetic semiconductor
}

\author{
M. C. Morón ${ }^{1}$ and S. Hull ${ }^{2}$ \\ ${ }^{1}$ Instituto de Ciencia de Materiales de Aragon, C.S.I.C.-Universidad de Zaragoza, E-50009 Zaragoza, Spain \\ ${ }^{2}$ The ISIS Facility, Rutherford Appleton Laboratory, Didcot, Oxfordshire OX11 0QX, United Kingdom
}

(Received 28 September 2001; published 20 November 2001)

\begin{abstract}
The controlled substitution of $\mathrm{Mn}$ by $\mathrm{Zn}$ within the diluted magnetic semiconductor $\mathrm{Zn}_{1-x} \mathrm{Mn}_{x} \mathrm{Ga}_{2} \mathrm{Se}_{4}(0$ $\leqslant x \leqslant 1$ ) series induces a pumping of magnetic ions from a defined site of the crystal lattice to another one driving the system to an order-disorder phase transition with $x_{c}=0.50 \pm 0.01$. A long-range order parameter, $\eta$, is defined and directly determined as a function of $x$ within the whole range of composition from neutrondiffraction experiments. An augmentation of the magnetic exchange field by increasing the magnetic dilution occurs as a consequence of that order-disorder process.
\end{abstract}

DOI: 10.1103/PhysRevB.64.220402

PACS number(s): 75.50.Pp, 64.70.Kb, 61.12.Ld

Diluted magnetic semiconductors (DMS's) are semiconductor compounds in which a fraction of nonmagnetic cations has been substituted by a magnetic transition metal. The interaction between the localized magnetic moments and the conduction and/or valence band electrons results in an unusual combination of electronic, optical, and magnetic properties that make these materials interesting subjects for scientific investigation and potential device applications. ${ }^{1}$ Through the use of solid solutions, the properties of DMS's can be tailored by varying the composition to precisely match specific requirements. ${ }^{2}$ However, one of the problems encountered in most of the bulk DMS's is the absence of complete solid solubility ${ }^{3}$ which restricts the experimental access to certain ranges of $x$ values.

Order-disorder phenomena in semiconductor alloys are processes with interesting theoretical implications and considerable technological importance. ${ }^{4}$ One reason that ordering has received so much attention is that the band-gap energy and other electronic and optical properties are strongly dependent on the order parameter. ${ }^{5}$ A quantitative determination of the degree of ordering is therefore particularly valuable. In addition to the classical pseudobinaries, ${ }^{6}$ experimental attempts to obtain new semiconductor alloys to explore ordering has led to the study of the family of chalcopyrites $A B C_{2} \cdot{ }^{7}$ The related family $A B_{2} C_{4}$, which is characterized by an ordered sublattice of vacancies and then by a further degree of freedom as to tailoring possibilities, has on the other hand received much less attention probably due to its higher degree of complexity. ${ }^{8}$

In this paper we present clear evidence of the occurrence of an order-disorder phase transition in a DMS series, $\mathrm{Zn}_{1-x} \mathrm{Mn}_{x} \mathrm{Ga}_{2} \mathrm{Se}_{4}$, as a function of the degree of dilution $x$ within the whole compositional range. The direct determination of a long-range order parameter, $\eta$, obtained for a DMS as a function of $0 \leqslant x \leqslant 1$ is presented from neutrondiffraction data. The fingerprint of that order-disorder transition and of $\eta$ on the magnetic properties of that series is also analyzed with the finding of additional phenomenology.

Orange single crystals of $\mathrm{Zn}_{1-x} \mathrm{Mn}_{x} \mathrm{Ga}_{2} \mathrm{Se}_{4}$ with $x$ $=0.00,0.104(4), 0.240(4), 0.343(4), 0.482(4), 0.63(1)$, $0.77(1), 0.900(7)$, and 1.00 were prepared at the Instituto de
Ciencia de Materiales de Aragon using the chemical vapor transport method $(\mathrm{CVT})$ at $800{ }^{\circ} \mathrm{C}$. ${ }^{9}$ The quartz ampoule was cooled to room temperature by carefully pulling it out of the furnace kept at that temperature. Time-of-flight neutron diffraction experiments were performed on the high-intensity powder diffractometer Polaris at the ISIS facility, UK. ${ }^{10}$ Diffraction data were collected at room temperature over the $d$-spacing range $0.5<d(\AA)<3.2$. The crystal structure of the series, including lattice site occupancies, was obtained by using the Rietveld refinement program TF14LS. ${ }^{11}$ The sum of the Mn site occupancy factors (see below for details) gives the total Mn concentration $x$ of each of the members of the series as given above. dc magnetic susceptibility data were taken on a magnetometer with superconducting quantum interference device detection manufactured by Quantum Design. The measurements were performed between 5 and 300 $\mathrm{K}$ under an external magnetic field of $1 \mathrm{~T}$ on polycrystalline samples of $\mathrm{Zn}_{1-x} \mathrm{Mn}_{x} \mathrm{Ga}_{2} \mathrm{Se}_{4}(0 \leqslant x \leqslant 1)$. The degree of dilution $x$ has been also calculated by applying the Curie-Weiss law to the high-temperature data $(130-250 \mathrm{~K})$. The obtained values coincide, within the experimental error, with those found from the structural data.

The crystal structures of the two end members of the series, $\mathrm{ZnGa}_{2} \mathrm{Se}_{4}$ and $\mathrm{MnGa}_{2} \mathrm{Se}_{4}$, were taken as starting points for determining the structural arrangement of the rest of the members of the series. At ambient conditions $\mathrm{MnGa}_{2} \mathrm{Se}_{4}$ possesses a defective chalcopyrite structure, space group $I-4$, with $a=5.677(1) \AA$ and $c=10.761(2) \AA$ and a completely ordered cation distribution [see Fig. 1(a)]. ${ }^{12}$ However $\mathrm{ZnGa}_{2} \mathrm{Se}_{4}$ exhibits a defective stannite structure, space group $I-42 m$, with a partial disordering of the cations and $a$ $=5.5117(3) \AA$ and $c=10.9643(6) \AA$ [see Fig. 1(b) $].{ }^{13}$ Both structures are defective with the vacancies located at the 2 (b) crystallographic position (see Fig. 1). One of the two gallium atoms of the unit formula occupies the same lattice site, $2(a)$, in both $\mathrm{ZnGa}_{2} \mathrm{Se}_{4}$ and $\mathrm{MnGa}_{2} \mathrm{Se}_{4}$. Concerning the cation distribution, the main difference between these two compounds is that in $\mathrm{MnGa}_{2} \mathrm{Se}_{4}$ the $\mathrm{Mn}$ atoms and half of the $\mathrm{Ga}$ atoms are placed in different crystallographic positions, $2(d)$ and 2(c) respectively, while in $\mathrm{ZnGa}_{2} \mathrm{Se}_{4}$ the $\mathrm{Zn}$ and half of the Ga atoms share the same crystallographic 


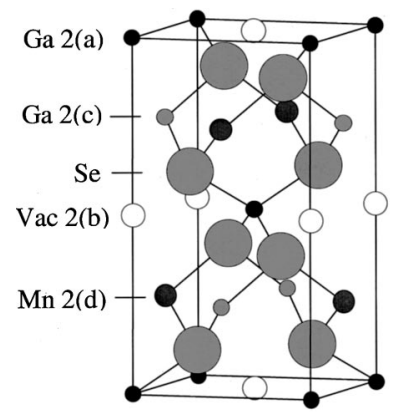

(a)

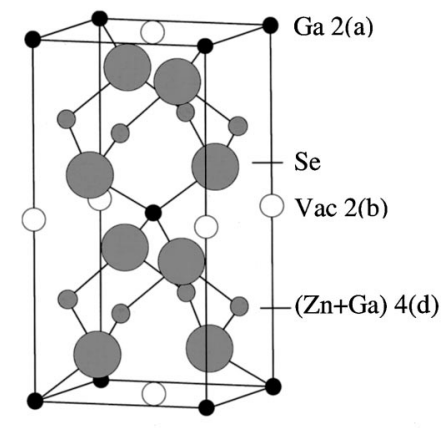

(b)
FIG. 1. Unit cells of $\mathrm{MnGa}_{2} \mathrm{Se}_{4}$ (a), space group I-4, and $\mathrm{ZnGa}_{2} \mathrm{Se}_{4}$ (b), space group $I-42 \mathrm{~m}$. The coordinates of relevant sites are 2(a): $(0,0,0,) ; \quad 2(b): \quad\left(0,0, \frac{1}{2}\right) ; \quad 2(c): \quad\left(0, \frac{1}{2}, \frac{1}{4}\right)$; $2(d): \quad\left(0, \frac{1}{2}, \frac{3}{4}\right) ; 4(d): \quad\left(0, \frac{1}{2}, \frac{1}{4}\right.$ and $\left.0, \frac{1}{2}, \frac{3}{4}\right)$. Vac stands for vacancy.

position, 4(d) (see Fig. 1). In fact, the lattice positions associated with $2(d)$ plus those corresponding to $2(c)$ in $I-4$ are those associated with $4(d)$ in $I-24 m$.

Full Rietveld structure refinements performed on the powder diffraction patterns reveal the symmetry and structural characteristics of the series. Thus I-4 is found to be the space group which better describes the crystal symmetry of $x$ $=1.00,0.90,0.77$, and 0.63 while $I-42 \mathrm{~m}$ is found to be the best choice for $x=0.48,0.34,0.24,0.10$, and 0.00 . The relevant criteria for choosing between space groups $I-4$ and $I-42 m$ has been the values of the $X$ and $Y$ positional coordinates of the Se atom. These two coordinates are equal in $I-42 m$, for symmetry reasons, but can take different values in the case of $I-4$. Thus a crossover from a $I-4$ to a $I-42 m$ symmetry occurs at intermediate values of the degree of dilution $x$ when varying the Mn concentration from $x=1$ to $x=0$. Atomic coordinates, unit cell values, isotropic temperature factors, and some related crystallographic information will be published elsewhere together with further details of the refinements. ${ }^{9}$

The distribution of the Mn cations in the lattice is presented in Fig. 2 as a function of the degree of dilution $x$. In the case of $x=1$, the Mn atoms exhibit a completely ordered scheme, all of them being placed at a well-defined crystallographic position $2(d)$. As the $x$ parameter decreases a pumping of magnetic ions from $2(d)$ to a nonequivalent crystallographic site 2(c) starts (see Fig. 1). As $x$ continues decreasing that pumping effect raises the $x^{(c)} / x^{(d)}$ ratio corresponding to the percentage of $\mathrm{Mn}$ on the $2(c)$ and $2(d)$ sites. At intermediate values of $x$ the probabilities of a $\mathrm{Mn}$ ion occupying the $2(d)$ and $2(c)$ sites become equal and the two sites come to be crystallographically equivalent $[4(d)$ site in space group $I-42 m]$. This situation is maintained as $x$ decreases down to $x=0$. Considering the distribution of the magnetic ions in the lattice, an order-disorder phase transition takes place within the $\mathrm{Zn}_{1-x} \mathrm{Mn}_{x} \mathrm{Ga}_{2} \mathrm{Se}_{4}$ series as a function of the concentration of Mn. A critical point $x_{c}=0.50$ \pm 0.01 can be deduced from Fig. 2. Since the Rietveld refinements also show that, within the whole range of $x$, no $\mathrm{Mn}$ is located on the 2( $a)$ and 2(b) sites (corresponding Mn site occupancy factors equal to zero), the total Mn concentration of each member of the series is obtained from the sum of the

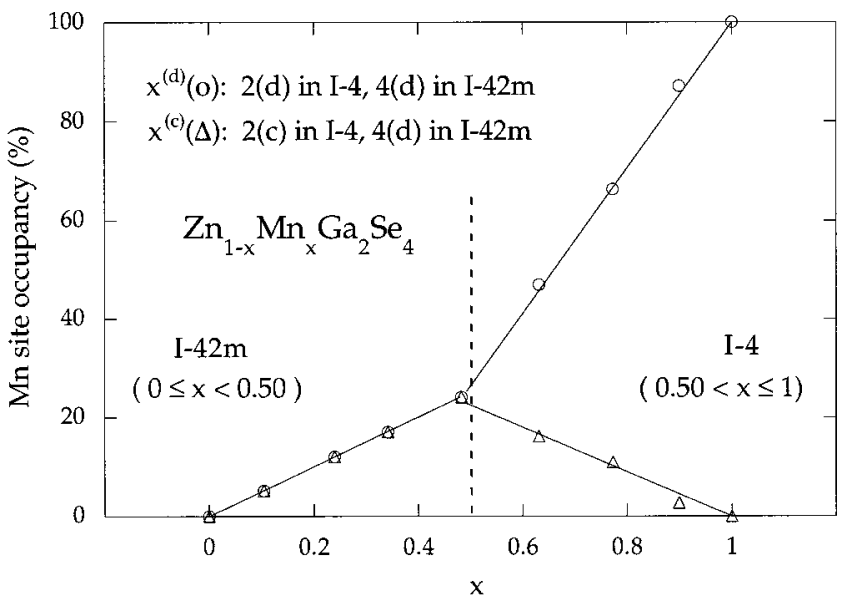

FIG. 2. Distribution of the Mn ions on the crystal lattice as a function of the concentration of the magnetic ion in the $\mathrm{Zn}_{1-x} \mathrm{Mn}_{x} \mathrm{Ga}_{2} \mathrm{Se}_{4}$ series. The dashed line separates areas of existence of space groups $I-42 \mathrm{~m}$ and $I-4$ (critical point at $x_{C}=0.50$ $\pm 0.01)$. The error in the occupancy is calculated to be less than $1 \%$.

$x^{(d)}$ and $x^{(c)}$ values shown in Fig. 2. The distribution of the $\mathrm{Zn}$ atoms, which share crystallographic positions with the Mn ions, also depends on $x$ and will be published elsewhere. ${ }^{9}$

The gradual change in crystal symmetry within the $\mathrm{Zn}_{1-x} \mathrm{Mn}_{x} \mathrm{Ga}_{2} \mathrm{Se}_{4}$ series would suggest a second-order nature of the order-disorder transition. In fact, $I-4$ is a subgroup of $I-42 m$. We have chosen the quantity $\eta=\left[x^{(d)}-x^{(c)}\right] /\left[x^{(d)}\right.$ $\left.+x^{(c)}\right]$ as a long-range order parameter ${ }^{14}$ describing the order-disorder phase transition exhibited by the series under study. The dependence of $\eta$ on $x$ is presented in Fig. 3. This order parameter fulfills the requirement of being equal to unity in the perfectly ordered state, $x=1$, and zero in the disordered state, $0 \leqslant x<0.5$. Therefore the degree of ordering of that series can be tuned from its minimum to its maximum values just by tailoring the composition. This is interesting since the degree of ordering has been shown to strongly influence some physical properties of semiconductors. ${ }^{5}$ In fact, the band gap of the $\mathrm{Zn}_{1-x} \mathrm{Mn}_{x} \mathrm{Ga}_{2} \mathrm{Se}_{4}$ series can be tuned by tailoring $\eta$ through $x$. $^{9}$

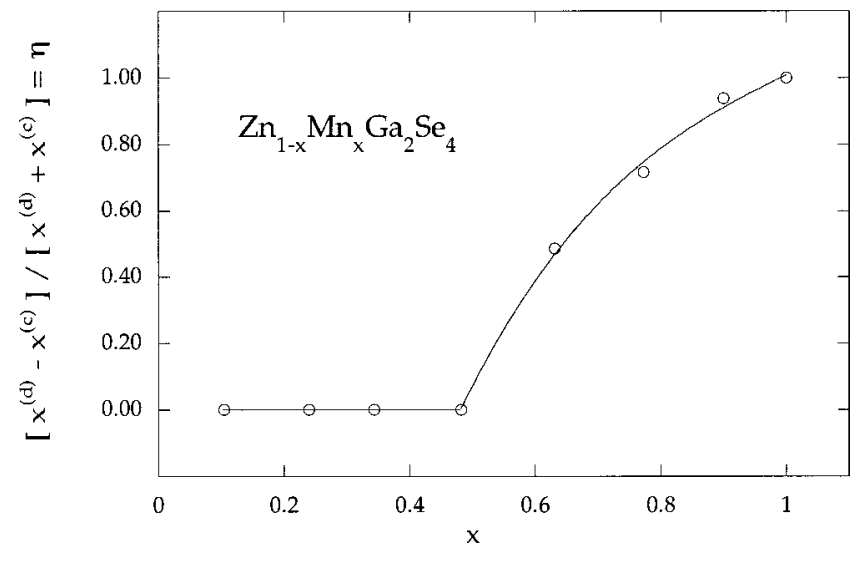

FIG. 3. Dependence of the long-range order parameter $\eta$ $=\left[x^{(d)}-x^{(c)}\right] /\left[x^{(d)}+x^{(c)}\right]$ on the concentration of $\mathrm{Mn}$ ion in the $\mathrm{Zn}_{1-x} \mathrm{Mn}_{x} \mathrm{Ga}_{2} \mathrm{Se}_{4}$ series. 


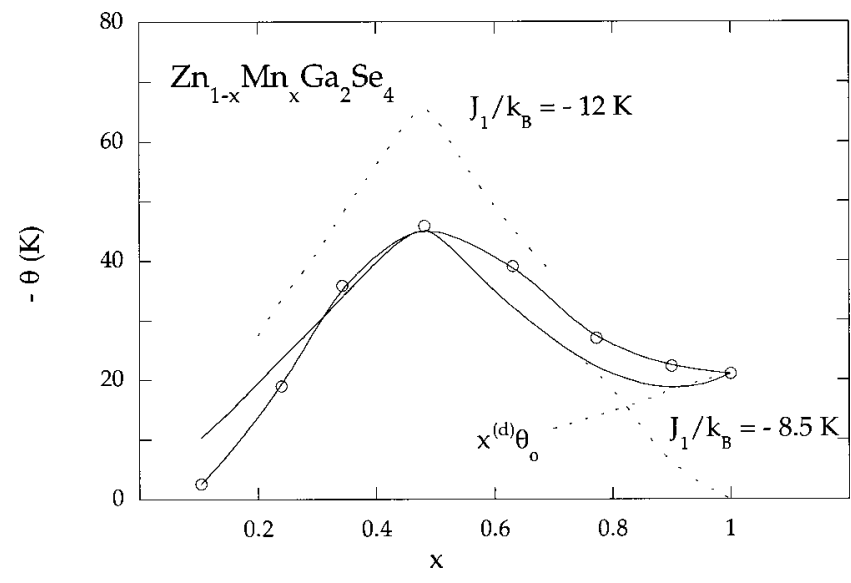

FIG. 4. Experimental (dots) and simulated (lines) Curie-Weiss temperature of the $\mathrm{Zn}_{1-x} \mathrm{Mn}_{x} \mathrm{Ga}_{2} \mathrm{Se}_{4}$ series as a function of $x$. The line connecting experimental points is an eye guide. Full line: $[2 S(S+1) / 3] 4 x^{(c)} J_{1} / k_{B}$ (named $\theta_{1}$ in the following) for $x<0.8$ and $x^{(d)} \theta_{0}$ for $x>0.8$, with $J_{1} / k_{B}=-8.5 \mathrm{~K}$ and $\theta_{0}=\theta(x=1)$. Upper broken line: $\theta_{1}$ with $J_{1} / k_{B}=-12 \mathrm{~K}$; lower broken lines: $\theta_{1}$ with $J_{1} / k_{B}=-8.5 \mathrm{~K}$ and $x^{(d)} \theta_{0}$. See text for details.

The particular distribution of Mn cations versus $x$ in $\mathrm{Zn}_{1-x} \mathrm{Mn}_{x} \mathrm{Ga}_{2} \mathrm{Se}_{4}$ also has a remarkable influence on the magnetic properties of the series. Thus the evolution of the Curie-Weiss temperature, $\theta$, with $x(0 \leqslant x \leqslant 1)$ is shown in Fig. 4. The negative sign indicates predominant antiferromagnetic interactions. These results have been calculated in the temperature range $130-250 \mathrm{~K}$, that is from data at $T$ $\gtrsim 5|\theta|$. Pseudobinary DMS's exhibit comparatively higher $|\theta|$ values $^{15}$ that makes the calculation of reliable CurieWeiss temperatures more difficult due to experimental limitations in the available temperature range. Superexchange has been found to be the dominant mechanism responsible for the Mn-Mn interaction in DMS containing that ion., ${ }^{3,16}$ The diamagnetic correction of the susceptibility data of the $\mathrm{Zn}_{1-x} \mathrm{Mn}_{x} \mathrm{Ga}_{2} \mathrm{Se}_{4}$ series does not change the value of the Curie-Weiss temperatures outside the experimental error. A clear maximum in $\theta$ can be observed at around $x=0.5$ (see Fig. 4) which interestingly coincides with the critical concentration, $x_{c}$, corresponding to the order-disorder phase transition. In the bibliography related to DMS's no evolution of $\theta$ with $x$ has been calculated within the whole range of concentration exhibiting such a maximum. Curie-Weiss temperatures are typically reported in a limited range of composition showing a decrease of $\theta$ with the concentration of magnetic ion. ${ }^{17}$ Within the molecular field theory the Curie-Weiss temperature is a function of the $x$ parameter through the expression $\theta(x)=2 S(S+1) \sum z_{i}(x) J_{i}(x) / 3 k_{B}$ where $S$ represents the spin of the involved magnetic ion ( $S=\frac{5}{2}$ in this case), the index of the summation refers to the magnetic superexchange pathways with $J \neq 0, z$ stands for the number of magnetic neighboring ions of a given one taken as reference, and $J$ represents the corresponding magnetic superexchange constant. An earlier work on the series under study reports a dependence of $\theta$ with the sample cooling rate used during the synthesis procedure. ${ }^{18}$ Thus, for slowly cooled samples, $\theta$ is nearly linear with $x$ but can be two or three times larger for rapidly quenched samples. The explanation given is a dependence of Mn cation ordering with cooling rate from high to room temperature. ${ }^{18-20}$ It is important to remark that the synthesis procedure is different in both cases: those works deal with powders obtained from the melt and we obtain single crystals from CVT.

Further analysis reveals the creation of a new magnetic superexchange pathway due to the pumping of magnetic cations from the $2(d)$ to the $2(c)$ sites. The new superexchange constant is expected to be much stronger than those reported for $\mathrm{MnGa}_{2} \mathrm{Se}_{4}$. Thus, when $x=1$ the magnetic ions are all in the same crystallographic positions, $2(d)$, and the magnetic superexchange pathways are of the type $\mathrm{Mn}-\mathrm{Se} \cdot \mathrm{Se}-\mathrm{Mn}$ with magnitudes of $J / k_{B}$ of the order of $0.1-0.5 \mathrm{~K} .^{19,21}$ However as soon as some $\mathrm{Mn}$ ions are promoted to the $2(c)$ site, as the dilution with $\mathrm{Zn}$ starts and $\eta$ departs from 1, magnetic contacts of the type Mn-Se-Mn appear (see Fig. 1) as those presented in the corresponding pseudobinary $\mathrm{Zn}_{1-x} \mathrm{Mn}_{x} \mathrm{Se}$ which exhibits a related crystal structure. ${ }^{3}$ The magnetic superexchange constant corresponding to $\mathrm{Mn}-\mathrm{Se}-\mathrm{Mn}$ pathways has been reported to be antiferromagnetic in character and of the order of $10 \mathrm{~K}$ in $\mathrm{Zn}_{1-x} \mathrm{Mn}_{x} \mathrm{Se}^{22,23}$ As $x$ decreases from $x=1$ to $x_{c}=0.5$ in $\mathrm{Zn}_{1-x} \mathrm{Mn}_{x} \mathrm{Ga}_{2} \mathrm{Se}_{4}$ the number of magnetic neighbors associated with the Mn-Se-Mn pathway, and therefore $|\theta|$, increases reaching a maximum value at intermediate values of $x$. From $x_{c}$ to $x=0$ the decrease in $|\theta|$ corresponds to a reduction of the concentration of magnetic ions.

In order to support this explanation, simulations of $\theta$ as a function of $x$ have been performed. The dependence of $[2 S(S+1) / 3] 4 x^{(c)} J_{1} / k_{B}$, named $\theta_{1}$ in the following, with $x$ is shown in Fig. 4 for $J_{1} / k_{B}=-12 \mathrm{~K}$ and $-8.5 \mathrm{~K}$ where $J_{1} / k_{B}$ and $\theta_{1}$ represent, respectively, the exchange constant and Curie-Weiss temperature corresponding to $\mathrm{Mn}-\mathrm{Se}-\mathrm{Mn}$ pathway. In the range of high $x$ concentration the exchange field created as a result of the pumping of magnetic cations from $2(d)$ to $2(c)$ sites, decreases to zero as $x$ increases to 1 . Therefore, in order to simulate the experimental values of $\theta$ of around $20 \mathrm{~K}$ in that $x$ range, magnetic contacts within the sublattice of corresponding $x^{(d)}$ population should be considered. Thus, the evolution of $x^{(d)} \theta_{0}$ with $x$ is shown in Fig. 4 where $\theta_{0}=\theta(x=1)$ represents the Curie-Weiss constant of the undiluted system $\mathrm{MnGa}_{2} \mathrm{Se}_{4} \cdot{ }^{17}$ Then, approximating $\theta(x)$ by $[2 S(S+1) / 3] 4 x^{(c)} J_{1} / k_{B}$ for $x<0.8$ and $x^{(d)} \theta_{0}$ for $x>0.8$, the tendency of the experimental data is impressively simulated when taking into consideration the simplicity of the model used: exchange constants between distant magnetic neighbors have been neglected, the expression for $\theta_{1}$ is strictly valid in the range $x^{(c)} \approx x^{(d)}$, the exchange constants are considered not to change significantly with $x$, and $x^{(c)}$ is neglected at high $x$ values. However, $J_{1} / k_{B}=-8.5 \mathrm{~K}$ should be regarded as an estimation of the order of magnitude of $J_{1} / k_{B}$ for $\mathrm{Zn}_{1-x} \mathrm{Mn}_{x} \mathrm{Ga}_{2} \mathrm{Se}_{4}$, rather than as an exact value, due to the approximations introduced in its estimation. Further detailed numerical simulations and experiments at low and high temperature are in progress.

We can conclude that the present study provides a clear demonstration of the existence of an order-disorder phase transition in a diluted magnetic semiconductor as a function 
of $x$, and, a direct determination in the whole range of composition of (i) the associated distribution of magnetic ions, and (ii) the corresponding long-range order parameter. Moreover an augmentation of $\theta$ by increasing the magnetic dilution is observed. The mechanism responsible for such behavior is found to be the occurrence of the order-disorder process. Thus, the pumping of magnetic ions from one site to another in the crystal lattice, as soon as dilution starts, gives rise to an additional magnetic superexchange pathway which exhibits a particularly intense exchange constant.

This research was supported by DGA Grant No. P059/ 2000, CICYT Grant No. MAT2000-1388C0303, and the EU IHP program for ISIS neutrons (RB 8760).
${ }^{1}$ M. Jain, Diluted Magnetic Semiconductors (World Scientific, Singapore, 1991); W. Zaets and K. Ando, Appl. Phys. Lett. 77, 1593 (2000).

${ }^{2}$ R. Rupprecht, B. Müller, H. Pascher, I. Miotkowski, and A. K. Ramdas, Phys. Rev. B 58, 16123 (1998); N. Karar, S. Basu, R. Venkataraghavan, and B. M. Arora, J. Appl. Phys. 88, 924 (2000)

${ }^{3}$ F. K. Furdyna, J. Appl. Phys. 64, R29 (1988).

${ }^{4}$ A. Zunger, Mater. Res. Bull. 22, 20 (1997).

${ }^{5}$ Y. Zhang, A. Mascarenhas, S. Smith, J. F. Geisz, J. M. Olson, and M. Hanna, Phys. Rev. B 61, 9910 (2000); T. Suzuki, Mater. Res. Bull. 22, 33 (1997).

${ }^{6}$ T. S. Kuan, T. F. Kuech, W. I. Wang, and E. L. Wilkie, Phys. Rev. Lett. 54, 201 (1985); R. G. Dandrea, J. E. Bernard, S. H. Wei, and A. Zunger, ibid. 64, 36 (1990).

${ }^{7}$ D. S. Su and S. H. Wei, Appl. Phys. Lett. 74, 2483 (1999); C. Rincon, Phys. Rev. B 45, 12716 (1992).

${ }^{8}$ V. V. Ursaki, I. I. Burlakov, I. M. Tiginyanu, Y. S. Raptis, E. Anastassakis, and A. Anedda, Phys. Rev. B 59, 257 (1999).

${ }^{9}$ M. C. Morón et al. (unpublished).

${ }^{10} \mathrm{~S}$. Hull and R. Smith, The ISIS Facility Word Wide Web, http://www.isis.rl.ac.uk.

${ }^{11}$ W. I. F. David, R. M. Ibberson, and J. C. Matthewman, Rutherford Appleton Laboratory, UK, Report No. RAL-92-032, 1992 (unpublished); P. J. Brown and J. C. Matthewman, Rutherford Appleton Laboratory, UK, Report No. RAL-87-010, 1987 (unpublished).
${ }^{12}$ M. Cannas, L. Garbato, A. G. Lehmann, N. Lampis, and F. Ledda, Cryst. Res. Technol. 33, 417 (1998).

${ }^{13}$ T. Hanada, F. Izumi, Y. Nakamura, O. Nittono, Q. Huang, and A. Santoro, Physica B 241-243, 373 (1998).

${ }^{14}$ C. N. R. Rao and K. J. Rao, Phase Transitions in Solids (McGraw-Hill, New York, 1978).

${ }^{15}$ F. K. Furdyna, N. Samarth, R. B. Frankel, and J. Spalek, Phys. Rev. B 37, 3707 (1988).

${ }^{16}$ H. Hass, in Semimagnetic Semiconductors and Diluted Magnetic Semiconductors (Plenum, New York, 1991).

${ }^{17}$ J. Spalek, A. Lewicki, Z. Tarnawski, J. K. Furdyna, R. R. Galazka, and Z. Obuszko, Phys. Rev. B 37, 411 (1988).

${ }^{18}$ J. C. Wooley, S. Bass, A. M. Lamarche, G. Lamarche, M. Quintero, M. Morocoima, and P. Bocaranda, J. Magn. Magn. Mater. 150, 353 (1995).

${ }^{19}$ J. C. Wooley, S. Bass, A. M. Lamarche, and G. Lamarche, J. Magn. Magn. Mater. 131, 199 (1994).

${ }^{20}$ J. C. Wooley, R. Brun del Re, and M. Quintero, Phys. Status Solidi A 159, 361 (1997).

${ }^{21}$ R. Rimet, C. Schlenker, and D. Fruchart, J. Phys. (France) 43, 1759 (1982).

${ }^{22}$ Y. Shapira, in Semimagnetic Semiconductors and Diluted Magnetic Semiconductors (Plenum, New York, 1991).

${ }^{23}$ V. Bindilatti, E. ter Haar, N. F. Oliveira, Y. Shapira, and M. T. Liu, Phys. Rev. Lett. 80, 5425 (1998). 